\title{
Preparation of PVDF-CNT composite
}

\author{
Denis Misiurev ${ }^{1}$, Ştefan Ţălu ${ }^{2, *}$, Rashid Dallaev ${ }^{1}$, Dinara Sobola ${ }^{1,3,4}$ and Mariya \\ Goncharova $^{5}$ \\ ${ }^{1}$ Brno University of Technology, Technická 2848/8, 61600 Brno, Czech Republic \\ ${ }^{2}$ The Technical University of Cluj-Napoca, Constantin Daicoviciu Street, no. 15, Cluj-Napoca, \\ 400020, Cluj county, Romania \\ ${ }^{3}$ Central European Institute of Technology BUT, Purkyňova 123, 61200 Brno, Czech Republic \\ ${ }^{4}$ Dagestan State University, Makhachkala, St. M. Gadjieva 43-a, Dagestan Republic 367015, Russia \\ ${ }^{5}$ Ural State University of Economics, Russian Federation
}

\begin{abstract}
Limitations of ceramic piezomaterials (brittleness, toxicity of lead-containing samples, difficulties of complicated shapes preparations, etc.) call for the research in the field of piezoelectric polymers. One of them is polyvinylidene fluoride (PVDF). It could be prepared in various forms: thin films, bulk samples, fibers. PVDF fibers attract the most attention because of high flexibility, lightweight, mechanical stability, chemical inertness. Properties of PVDF fibers can be tuned using dopant material: ceramic particles, metal nanoparticles, graphite materials as graphene oxide or carbon nanotubes (CNT).
\end{abstract}

\section{Introduction}

Poly (vinylidene fluoride), PVDF and its copolymers are a class of high-performance polymers that have the highest dielectric constant and electroactive response, as well as piezoelectric, pyroelectric and ferroelectric effects.

Electroactive properties are applied in various fields, such as biomedicine, energy generation and storage, monitoring and control, and include the development of sensors and actuators, separating and filtering membranes and intelligent scaffolding (Martins et al. 2014).

Polyvinylidene fluoride (PVDF) is a semi-crystalline, non-reactive high purity thermoplastic fluoropolymer that is approximately fifty percent amorphous. The percentage of crystallinity is based on the chain ordering defects. PVDF is readily melt-processible and can be fabricated into parts by injection and compression molding (Martins et al., 2014; Li et al., 2019).

Thanks to its excellent combination of properties and processability, PVDF has become the largest volume of fluoropolymers after PTFE. PVDF is available commercially in a wide range of melt flow rates and with various additives to enhance processing or end use properties.

\footnotetext{
* Corresponding author: stefan ta@yahoo.com
} 
Well-characterized properties make the PVDF leader of piezoelectric polymers. It can boast extraordinary mechanicals and chemical parameters such as: deformation resistance, absorption resistance, chemical resistance, stability to radiation, high working temperature (from $-49^{\circ}$ to $302{ }^{\circ} \mathrm{F}$ ), insulating capabilites; radiation stability, high Curie point (217.4 ${ }^{\circ} \mathrm{F}$ ), high degree of purity. Such combination of properties makes PVDF useful for varieties of application where the polymer is used in: aerospace, biosensors, biotechnologies, pharmaceutical, microelectronics, pressure sensors, insulator for batteries.

In electronics industry PVDF is used especially as jacketing materials for plenum- rated cable used in voice and video devices and alarm systems.

The low flame spread and smoke generation of PVDF is a prime asset in these applications. Recently emerging applications of PVDF include fuel cell membranes, and components for aircraft.

The atomic structure of PVDF is represented by monomer $-\mathrm{CH}_{2} \mathrm{CF}_{2}-$. The molecular weight of the monomer is between 16 and $17 \mathrm{~kg} / \mathrm{mol}$.

The monomer $-\mathrm{CH}_{2} \mathrm{CF}_{2}-$ has strong electrical dipole moment with regards to electronegativity of fluorine atoms. The monomer forms chains with perpendicular orientation of dipole moments. The PVDF's properties with most polymers has been intensively studied (Correia et al., 2005; Imamura et al., 2008; Gomes et al., 2010; Patro et al., 2010; Koseki et al., 2012; Mandal et al., 2012; Cardoso et al., 2012; Zhu et al., 2014; Ruan et al., 2018).

The main focus of research has been done on two most important aspects, the first is polymorphism and second is piezoelectronic respond (Li et al., 2013).

PVDF is semicrystalline material crystallized into five different crystal phases: $\alpha, \beta, \gamma$, $\delta, \varepsilon$ obtained by different process parameters (You et al., 2017). The most stable at room temperature $\alpha$ phase is commonly synthesized among other phases, although the $\alpha$ phase is electrically neutral (non-polar), because of its antiparallel dipoles' alignment. After applying mechanical stretching of alignment, high pressure, changing temperature of crystallization of melt the $\alpha$ phase can be transferred into oriented $\beta$ phase (Li et al., 2013).

The $\beta$ phase is the most important due to its superior electrical, ferro-electrical and pyro-electrical properties in compare to other phases. Has been found the $\beta$ phase not only represents huge potential due to the strongest piezoelectric respond among all PVDF's phases, but also the PVDF itself has the strongest piezoelectric response amongst all commercial polymers.

Carbon nanotubes are unique carbon fibers with similar structure of fullerene. As other unique properties have been discovered such as remarkable mechanical and electronic properties unique Raman spectra, thermal conductivity, toughness, interest to nanotubes started to grow and their potential use in wide varieties of applications especially in nanodimension electronics and medicine (Kennedy et al., 2016, Knápek et al., 2017, Knápek et al., 2019, Knápek et al., 2020, Sobola et al., 2020).

An ideal nanotube represents hexagonal network of carbon atoms rolled up to form seamless cylinder. Base on amount layers, nanotubes are subdivided on two types: single-walled nanotubes (SWNT) and multi-wall nanotubes (MWNT) (Mirjalili and Zohoori, 2016).

The composite materials can be characterized by different spectroscopic and microscopic techniques such as XRD, FTIR, thermal and FE-SEM analyses (Țălu, 2015; Ţălu et al., 2018; Mwema et al., 2020).

\section{Experimental data}

\subsection{Preparation of CNT dopant}


The Plasma Enhanced CVD (Chemical Vapor Deposition) is a variation of the CVD, where inert gas plasma is used to produce thin films. The technique has been developed to produce not only wide variety of organic and inorganic films but also Multi-Wall Nanotubes of high quality, which do not require additional purification from by-products (Mirjalili and Zohoori, 2016; Bin et al., 2018). The SEM images for the nanotubes prepared in this study are given in Figure 1, 2(a, b).

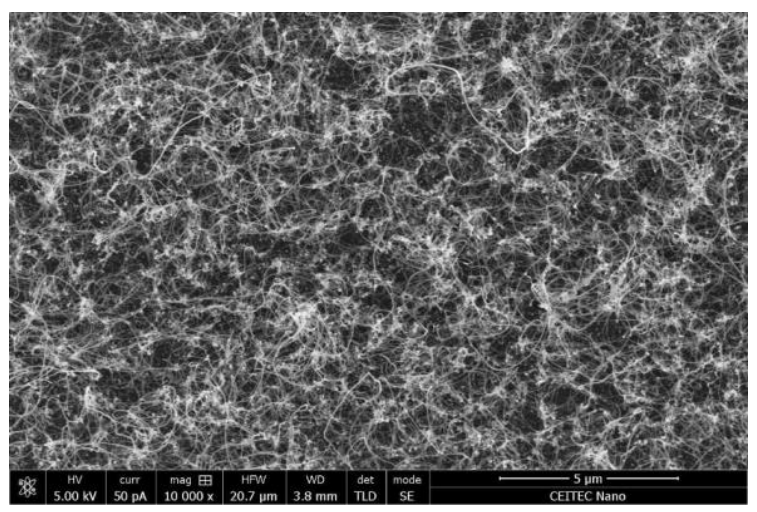

Fig. 1. SEM image of the produced nanotubes.

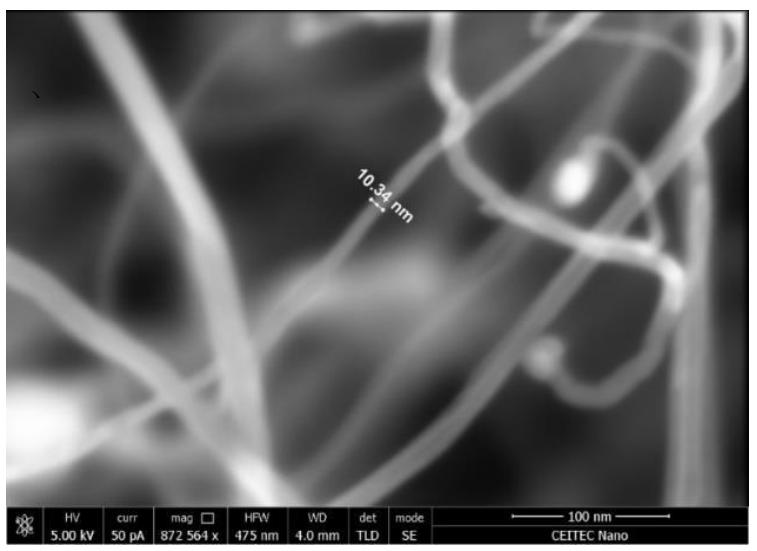

a)

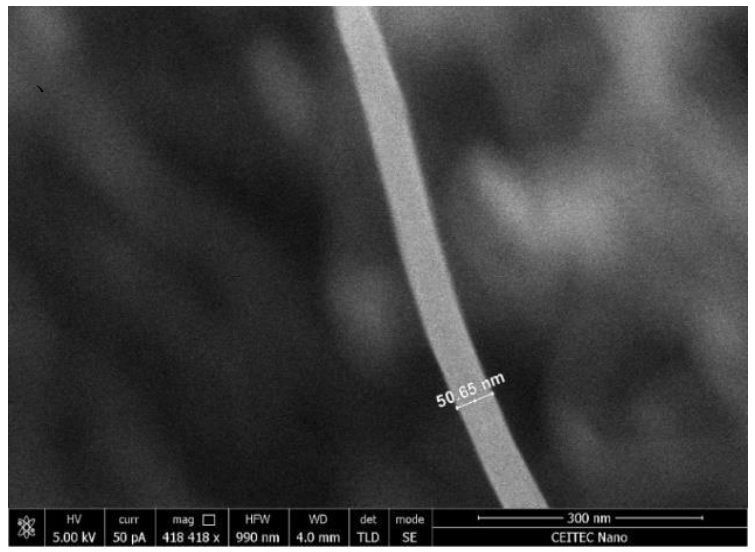

b) 
Fig.2. a, b) Different diameter of produced nanotubes.

Multi-Wall Nanotubes has been produced on $5 \mathrm{~nm}$ thick iron film silicon substrates and according to followed recipe:

1) Pumping for 30 seconds.

Main chamber of the reactor must be cleaned from any particles to avoid possible contamination resulted nanotubes.

2) Heat up to $750{ }^{\circ} \mathrm{C}, 1000 \mathrm{mTorr}$ (Torr is $133.3224 \mathrm{~Pa}$ ), Ar $1000 \mathrm{sccm}$ (Standard cubic centimeters per minute), transfer sample into the main chamber at $695^{\circ} \mathrm{C}$.

Main chamber needs to heat up to increase reactivity of poring chemicals and create appropriate pressure in it. Substrates must be interred into main chamber heated to avoid heat stress with presence or inert atmosphere.

3) $\mathrm{H}_{2} \mathrm{Stab}, 750^{\circ} \mathrm{C}, 1 \mathrm{~min}, 1000 \mathrm{mTorr}, \mathrm{H}_{2} 200 \mathrm{sccm}$.

Poring certain amount of hydrogen into main reactor at same temperature as main reactor under defined pressure.

4) $\mathrm{H}_{2}$ pretreatment, $750{ }^{\circ} \mathrm{C}, 10 \mathrm{~min}, 1000 \mathrm{mTorr}$ Ar $1000 \mathrm{sccm}+\mathrm{H}_{2} 200 \mathrm{sccm}$

Mixing hydrogen with inert atmosphere.

5) Growth, $750{ }^{\circ} \mathrm{C}, 30 \mathrm{~min}, 1000 \mathrm{mTorr}, \mathrm{Ar} 1000 \mathrm{sccm}+\mathrm{H}_{2} 200 \mathrm{sccm}+\mathrm{C}_{2} \mathrm{H}_{2} 20 \mathrm{sccm}$

Initiation of growing process with presence of hydrocarbon gas mixed with hydrogen.

6) Cooling down, 1000 mTorr, Ar $1000 \mathrm{sccm}$, transfer to loadlock $<695{ }^{\circ} \mathrm{C}$, after that cooling down without Ar, 1000 mTorr.

Hot substrates are transferred back into loadlock and cooling down without present of inert gases.

\subsection{Electrospinning of Polyvinylidene Fluoride}

There are many available commercial technologies of producing Nanofibers, however there is one technology which allow produce fine fibers ( $\mathrm{Li}$ et al., 2019).

Electrospinning is unique self-assembly technology offers wide variety of adjustable parameters to produce required fibers. Electrospinning is the thirst technology allowed to produce fibers which formation is given by electrostatic forces rather mechanical. Fibers obtained by using electrospinning are self-organizable (Li et al., 2013).

Producing huge range of nanofibers and controlling their morphology by changing process parameters the electrospinning gained huge popularity and widely used in nowadays (You et al., 2017).

The basic setup is consisted of two counter electrodes and DC voltage supply applied on needle-based electrode. One of the counter electrodes is represented by metallic needle, the second, however, can be different one based on application the fibers are produced for. The most popular is cylinder counter electrode because of its simplicity. To simplify cleaning counter electrode and removing produced fibers the counter electrode is covered by foil of conductive material.

The Aluminum foil is one the most spreader conductive material is used for electrospinning. In order to transport precursor (melted polymer) the certain approach is required ( $\mathrm{Li}$ et al., 2013). It has been found experimentally a tiny, silicon tube and commercial, medical syringe are perfect to transport precursor into metallic needle and then start the spinning of nanofibers (Kennedy et al., 2016). Figure 3 illustrates different varieties of counter electrodes. 

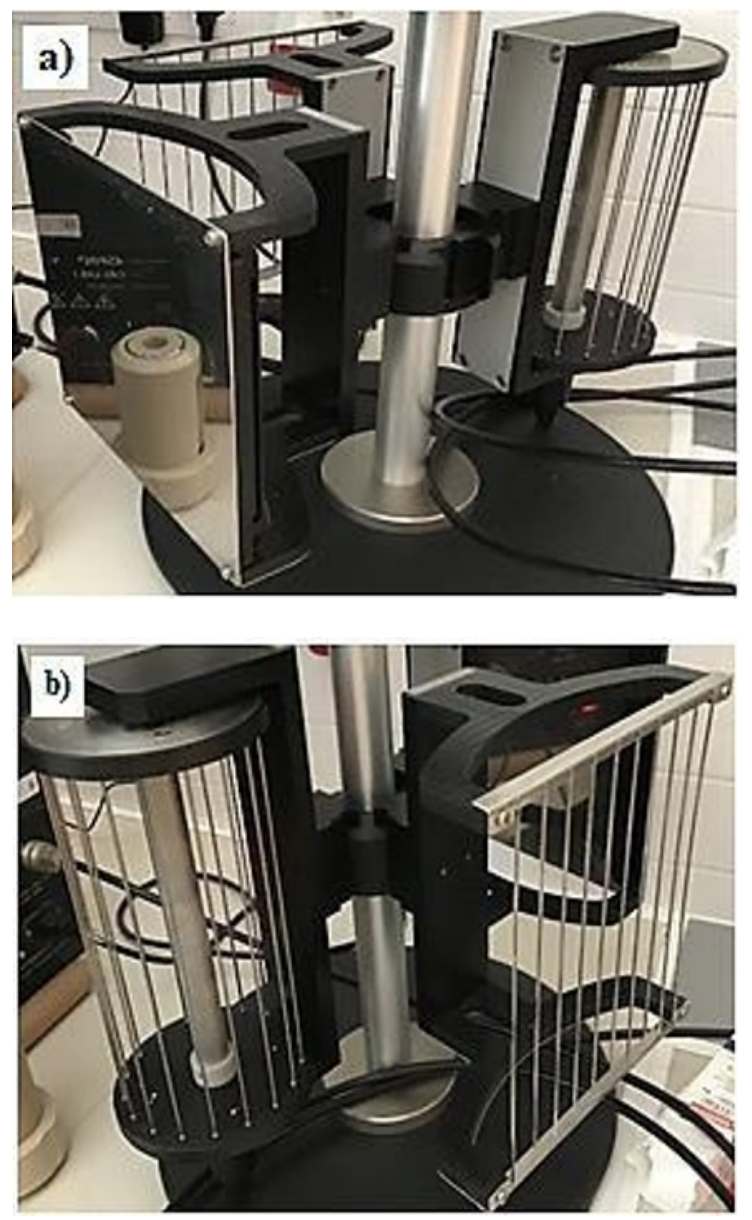

Fig. 3. a, b) Different varieties of counter electrodes.

To annihilate the process itself is needed to set up working parameters according to corresponding recipe of polymer to make fibers from.

Parameters in most cases are given as followed:

- Working Distance between counter electrodes: 8-20 cm

- Applied DC voltage: 0-60 kV

- Polarity of electrostatic field: positive

- Ability to use Hot Air: Temperature: $50-100{ }^{\circ} \mathrm{C}$, air flow: 0-50 1/min

- Number of rotations: 0-3000

- Needle diameter: 0.1-1 mm

Hot Air mode is used to speed up drying process of solvent, thus it will help to avoid popular defect of commercial fiber - thickened drops of precursor. Hot Air can be essential in cases where precursor is rather old, badly spined, watery. Hot Air is used not only to speed up drying precursor solvent by lowering humidity and helps to avoid the most popular defect calls "thickened drops of precursor" but also improve quality and properties of produced fibers.

At the end of process, the Aluminum foil must be carefully removed from counter electrode to do not deform the foil and fibers themselves. Figure 4 demonstrate SEM image of defect calls thickened drops of precursor. 


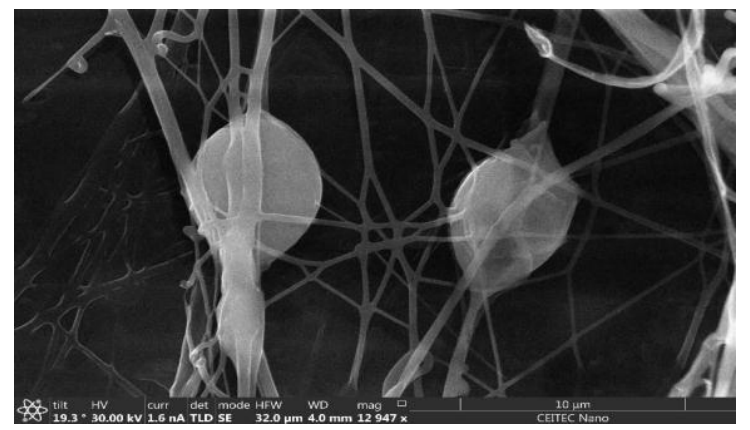

Fig. 4. Defect calls thickened drops of precursor.

The orientation of produced fibers is represented in two different ways: oriented and nonoriented. The orientation of produced fibers is given by amount of rotation per minute the fibers are collected on counter electrode. During the process, fibers formed within Taylor cone are collected on counter electrode. If the amount of rotation is not high enough produced fiber are suspended under the needle and slowly collected on counter electrode creating chaotically orientated thin film.

The Focused Ion Beam (FIB) technology offers a huge opportunity to study nano dimensional structures by using direct nanoscale and micro scale removal of studied material. FIB system combines both study of material cross section and imaging capabilities with high resolution (Fig. 5).

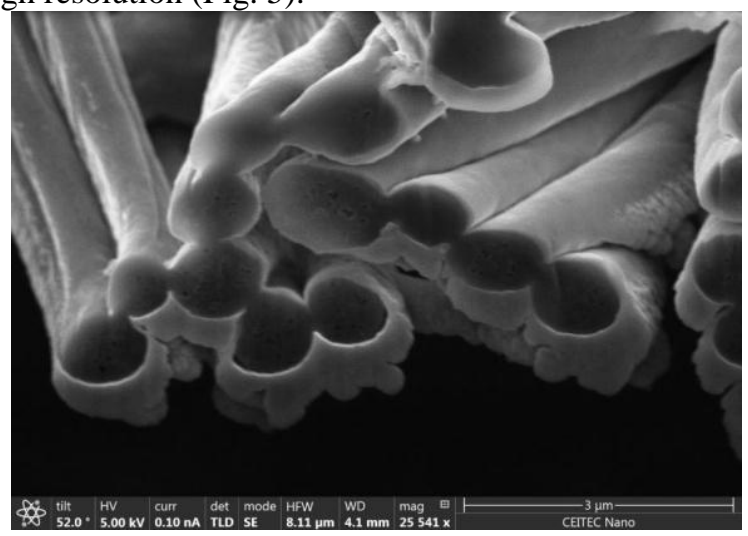

Fig. 5. Result of milling of cluster of fibers.

The samples have water-repellent and self-cleaning properties which makes them resistant to pollutions (Fig.6).

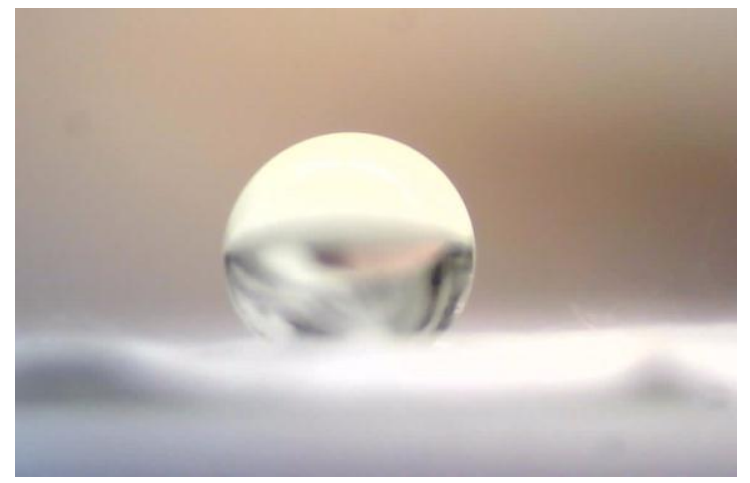


Fig. 6. Photo of water drops on the surface of the fibers.

\section{Conclusions}

The Polyvinylidene Fluoride polymer is unique material, which nowadays draws a huge amount of attention. Even though many aspects of the polymer have been studied extensively, there are still much more that requires further research, since full potential of Polyvinylidene Fluoride is yet to be revealed.

This material has found his place in many areas we all familiar with such as solar panels, batteries, alarm systems, medical masks, filters etc. however, the material has more to offer to all aspects of applied science, manufacture, and medicine.

The study also describes main aspects of potential addon to the Polyvinylidene Fluoride polymer, which will enhance its unique properties. This addon is called Carbon Nanotubes. Carbon Nanotube powder is rather expensive to use as an addon to Polyvinylidene Fluoride, however their unique properties such as conductivity, mechanical strength, chemical resistance is worth to use the nanotubes as a potential addon.

Research described in this paper was financially supported by Internal Grant Agency of Brno University of Technology [Grant No. FEKT-S-20-6352] and Grant Agency of Czech Republic under project No. 19-17457S. A part of the work was carried out with the support of CEITEC Nano Research Infrastructure [grant ID LM2015041, MEYS CR, 2016-2019], CEITEC Brno University of Technology.

\section{References}

1. D. Bin, W. Xianfeng, Y. Jianyong, Electro-spinning: Nanofabrication and Applications (Elsevier Science \& Technology Books, 2018)

2. V.F. Cardoso, C.M. Costa, G. Minas, S. Lanceros-Mendez, Smart Mater. Struct., 21 (2012)

3. H.M.G. Correia, M.M.D. Ramos, Comput. Mater. Sci., 33, 224 (2005)

4. J. Gomes, J. Serrado Nunes, V. Sencadas, S. Lanceros-Mendez, Smart Mater. Struct., 19 (2010)

5. R. Imamura, A.B. Silva, R. Gregorio, J. Appl. Polym. Sci. 110, 3242 (2008)

6. Y. Li, C. Liao, S.C. Tjong, Nanomaterials, 9, 952 (2019)

7. Y. Li, X. Ge, L. Wang, L. Wang, W. Liu, H. Li, R.Kwok, S. Chin, Curr. Nanosci., 9, 679 (2013)

8. K. Kennedy, A. Bhaw-Luximon, D. Jhurry, Acta Biomaterialia, 50, 41 (2016)

9. A. Knápek, D. Sobola, D. Burda, A. Danhel, M. Mousa, V. Kolarik, Nanomaterials, 9(12), 1756 (2019)

10. A. Knápek, D. Sobola, P. Tomanek, Z. Pokorna, M. Urbanek, Appl. Surf. Sci., 395, 157 (2017)

11. A. Knápek, R. Dallaev, D. Burda, D. Sobola, M.M. Allaham, M. Horaček, P. Kaspar, M. Matejka, M. Mousa, Nanomaterials, 10(7), 1294 (2020)

12. Y. Koseki, K.Aimi, S. Ando, Polym. J., 44, 757 (2012)

13. D. Mandal, K.J. Kim, J.S. Lee, Langmuir, 28, 10310 (2012)

14. P. Martins, A.C. Lopes, S. Lanceros-Méndez, Prog. Polym. Sci., 39, 683 (2014)

15. M. Mirjalili, S. Zohoori, J Nanostruct Chem., 6, 207 (2016) 
16. F.M. Mwema, E.T. Akinlabi, O.P. Oladijo, O.S. Fatoba, S.A. Akinlabi, Ş. Ţălu, Modern Manufacturing Processes, 1(1-2), 13 (2020)

17. T.U. Patro, M.V. Mhalgi, D.V. Khakhar, A. Misra, Polymer, 49, 3486 (2008)

18. L. Ruan, X. Yao, Y. Chang, L. Zhou, G. Qin, X. Zhang, Polymers, 10, 228 (2018)

19. D. Sobola, Sh. Ramazanov, M. Konečný, F. Orudzhev, P. Kaspar, N. Papež, A. Knápek, M. Potoček, Materials, 13(10), 2402 (2020)

20. Ş. Țălu, Micro and nanoscale characterization of three dimensional surfaces. Basics and applications (Napoca Star Publishing House, Cluj-Napoca, Romania, 2015)

21. Ş. Ţălu, D. Sobola, N. Papež, R. Dallaev, P. Sedlák, DEStech Transactions on Social Science, Education and Human Science, 202 (2018)

22. H. You, Z. Wu, Y. Jia, X. Xu, Y. Xia, Z. Han, Y. Wang, Chemosphere, 183, 528 (2017)

23. H. Zhu, S. Yamamoto, J. Matsui, T. Miyashita, M. Mitsuishi, J. Mater. Chem. C., 2, 6727 (2014) 